\title{
Read My Lips: An Animated Face Helps Communicate Musical Lyrics
}

Dominic W. Massaro and Miguel Hidalgo-Barnes

Perceptual Science Laboratory, University of California, Santa Cruz

Santa Cruz, CA. 95064 U.S.A.

\begin{abstract}
Understanding the lyrics of many contemporary songs is difficult. Watching the talker's face improves speech understanding when the speech is degraded by noise or hearing difficulty. To explore whether the face can be similarly helpful in music, 34 phrases from the song "The Pressman" by Primus (1993) were played to thirteen college students. These phrases were aligned with Baldi ${ }^{\circledR}{ }^{1}$, a computer-animated talking head. There were three presentation conditions: original audio, Baldi's mouthing of the lyrics, and the auditory lyrics aligned with Baldi. The students were asked to watch and listen and to type in as many words as they could understand. Performance was significantly better in the bimodal condition than the auditory condition, showing that visual information from the face contributes to the recognition of musical lyrics. The contribution of the face was somewhat small relative to that found in speech, however, and reasons for this difference remain to be determined.
\end{abstract}

\section{INTRODUCTION}

A variety of analogies have been drawn between language and music (e.g., Patel, 2003). Although somewhat limited in scope, both domains might be considered to be forms of communication. If we define linguistic and paralinguistic (or perhaps literal and nonliteral) dimensions of communication, we can claim that language emphasizes the literal relative to the paralinguistic whereas musical pieces emphasize the paralinguistic relative to the linguistic. In this research, we test whether the visual modality influences music perception in the same way that it does in spoken language perception.

It has been repeatedly shown that many things affect the understandability of verbal communication. In

\footnotetext{
${ }^{1}$ Baldi is a registered trademark of Dominic W. Massaro.
}

addition to the auditory and contextual information received by listening to a speaker, comprehension is aided visually, by being able to see the speaker's face while talking. This phenomenon is most evident in cases where the verbal information is degraded in some way, such as with noise or hearing impairment. Visual information from the face improves speech recognition and comprehension (Massaro, 1998).

\section{METHOD}

Participants. Thirteen students from the undergraduate Psychology participant pool at the University of California at Santa Cruz participated in this experiment.

Stimuli. The auditory component of this experiment consisted of a series of 34 one to three second verses from the song "The Pressman" by Primus (1993).

These samples were then viterbi aligned and hand adjusted such that Baldi visually portrayed each phoneme that occurred in each word of the lyrics at the same time and for the same duration as the auditory phonemes present in the song recording.

Procedure. In each of two sessions, the participant was shown each of the 34 samples once in each of the three modalities, Auditory only (A), Visual only (V), and both Auditory and Visual (AV), for a total of 102 trials. These 102 unique trials were randomly presented within the session.

\section{RESULTS}

The proportion of words correctly recognized regardless of position in the lyric was computed for each participant for each of the experimental conditions: session, verse, and presentation condition $(\mathrm{A}, \mathrm{V}, \mathrm{AV})$. An analysis of variance was carried out on the proportion of words correctly recognized (pooled across verse) as the dependent variable and the independent variables of session (first, second) and presentation condition (A, V, 
AV). The results show that the presence of the face did indeed help in lyric comprehension. The participants were able to understand $28 \%$ and $4 \%$ of the lyrics with just the auditory and visual lyrics, respectively, whereas performance was $33 \%$ in the bimodal presentation, $\mathrm{F}(2,24)=527, \mathrm{p}<.001$. A specific comparison between the $\mathrm{A}$ and $\mathrm{AV}$ conditions was statistically significant, $\mathrm{F}(1,12)=$ $52.9, \mathrm{p}<.001$.

\section{DISCUSSION}

The research demonstrated that a computeranimated face, Baldi, facilitated the recognition and short-term memory for immediate recall of a song's lyrics. Although the effect was highly significant, the improvement in performance of $28 \%$ to $33 \%$ was relatively small. The contribution of the face to the intelligibility of spoken sentences has been found to be much larger. In one study with 71 students (Jesse et al., 2000/01), the test items consisted of 65 meaningful sentences, e.g. "We will eat lunch out", presented in noise. When we computed the advantage given by Baldi across different levels of performance, there was an advantage of about $34 \%$ for participants at about the same level of performance as the participants in our current study.

The discrepancy might be due to differences in the advantage afforded by the face in speech and music conditions. To test this hypothesis, we repeated the experiment with the musical lyrics presented in a non-musical context. If the musical context was responsible for smaller visual advantage, then we would expect a larger visual effect. The original lyrics were viterbi aligned and hand adjusted for each phoneme that occurred in each word of the lyrics to obtain the location and duration of the phonemes in the sound sample. These phonemes and durations were then used as input to the Festival text-to-speech synthesis, which produced the auditory speech rendition of the lyrics. These same phonemes and durations were also used as input to our visible speech synthesis system used control Baldi's articulation in the visual and bimodal speech conditions. Baldi visually portrayed each phoneme that occurred in each word of the lyrics at the same time and for the same duration as the auditory phonemes present in the song recording.

The results of this new study showed that the presence of the face did indeed help in lyric comprehension, but the visual advantage was no larger than it was in the musical context.
Future studies will have to address why the sentences in noise benefited so much more by the presence of Baldi than did the musical lyrics in both a musical and non-musical context. A potentially important difference is that noise did not have to be added in the lyrics experiments because the lyrics were already difficult to recognize without noise.

Unfortunately, a visual alone condition was not included in the sentences in noise study as it was in the current music experiments. It remains possible that the visual speech in the sentences in noise study was much more informative than it was in the lyrics experiment and, therefore, the bimodal advantage would necessarily be larger in the sentences in noise study than in the lyrics experiment. We are currently testing this possibility, and the outcome of this experiment and its implications will be discussed.

Another issue is how much a real face synchronized with the musical lyrics would have improved performance. We expect that a real face would have given a larger improvement, as is the case in spoken language. In the same study with sentences in noise (Jesse et al., 2000/01), a real face gave about a 27\% improvement relative to Baldi's $21 \%$ improvement.

\section{REFERENCES}

[1]. Jesse, A., Vrignaud, N., \& Massaro, D. W. (2000/01). The processing of information from multiple sources in simultaneous interpreting. Interpreting, 5, 95-115.

[2]. Massaro, D. W. (1998). Perceiving talking faces: From speech perception to a behavioral principle. Cambridge, Massachusetts: MIT Press.

[3]. Patel A.D. (2003). Language, music, syntax and the brain. Nature Neuroscience 6(7):674-681.

[4]. Primus (1993). The Pressman. From the Album Pork Soda. Interscope Records.

\section{ACKNOWLEDGEMENT}

This work was supported in part by grants from the National Science Foundation (NSF Grant BCS9905176), a grant from the Public Health Service (Grant PHS R01 DC00236), cooperative grants from the Intel Corporation and the University of California Digital Media Program (D97-04), and grants from the University of California, Santa Cruz. The authors thank Ani Patel for helpful comments on this research and paper. Send correspondence to Dominic W. Massaro, Department of Psychology, University of California, Santa Cruz, CA 95064, USA. Electronic mail may be sent to massaro@fuzzy.ucsc.edu or infernarl@yahoo.com. 